\title{
Shaping the Design of Smartphone-Based Interventions for Self-Harm
}

\author{
Mahsa Honary \\ University of Cambridge \\ Cambridge, UK \\ mhonarykh@yahoo.com
}

\author{
Julio Vega \\ University of Manchester \\ Manchester, UK
}

\author{
Beth Bell \\ York St. John University \\ York, UK \\ b.bell@yorksj.ac.uk
}

\author{
Leo Kroll \\ Pennine Care Foundation Trust \\ Manchester, UK \\ Roisin McNaney \\ University of Bristol \\ Bristol, UK \\ r.mcnaney@bristol.ac.uk
}

\author{
Sarah Clinch \\ University of Manchester \\ Manchester, UK \\ sarah.clinch@manchester.ac.uk
}

\author{
Aaron Sefi Sefi \\ XenZone \\ London, UK
}

\begin{abstract}
Self-harm is a prevalent issue amongst young people, yet it is thought around $40 \%$ will never seek professional help due to stigma surrounding it. It is generally a way of coping with emotional distress and can have a range of triggers which are highly heterogeneous to the individual. In a move towards enhancing the accessibility of personalized interventions for selfharm, we undertook a three-stage study. We first conducted interviews with 4 counsellors in self-harm to understand how they clinically respond to self-harm triggers. We then ran a survey with 37 young people, to explore perceptions of mobile sensing, and current and future uses for smartphone-based interventions. Finally, we ran a workshop with 11 young people to further explore how a context-aware self-management application might be used to support them. We contribute an in-depth understanding of how triggers for self-harm might be identified and subsequently predicted and prevented using mobile-sensing technology.
\end{abstract}

\section{Author Keywords}

Self-harm; non-suicidal self-injury; mobile sensing; mental health; co-design; intervention; trust; situation-aware app.

\section{CCS Concepts}

-Human-centered computing $\rightarrow$ Human computer interaction (HCI); Haptic devices; User studies;

\footnotetext{
Permission to make digital or hard copies of all or part of this work for personal or classroom use is granted without fee provided that copies are not made or distributed for profit or commercial advantage and that copies bear this notice and the full citation on the first page. Copyrights for components of this work owned by others than the author(s) must be honored. Abstracting with credit is permitted. To copy otherwise, or republish, to post on servers or to redistribute to lists, requires prior specific permission and/or a fee. Request permissions from permissions@ acm.org.

CHI'20, April 25-30, 2020, Honolulu, HI, USA

(C) 2020 Copyright held by the owner/author(s). Publication rights licensed to ACM. ISBN 978-1-4503-6708-0/20/04 . .\$15.00

DOI: https : //doi .org/10.1145/3313831. XXXXXXX
}

\section{INTRODUCTION}

Self-harm refers to the deliberate injury of one's self (mainly characterised in mainstream media through cutting) [44]. It is particularly common among adolescents; with prevalence rates thought to be between $13-28 \%$ in non-clinical samples of adolescents [42]. The average age of onset for self-harm is around 12-14 years old [32] and adolescent girls are more likely to engage in self harm practices than adolescent boys [58]. It is estimated that $40 \%$ of the youth who self-harm will not report they do so or seek professional help, largely due to the stigma associated with self-harm, fears surrounding confidentiality and perceptions of negative reaction from others [55]. As a consequence, reported self-harm prevalence rates may underestimate the proportion of adolescents experiencing these negative thoughts and behaviours.

Self-harm is a major public health concern. However, due to the stigma surrounding self-harm, understanding and intervening in this issue is a challenge. Developing an enhanced understanding of the causes of self-harm during adolescence is of paramount importance, as too is the need to develop effective interventions which are appropriate to adolescents.

Current research in supporting young people with self-harm experiences is mainly focused on creating self-help tools (e.g. web-based and mobile apps) based on a wide range of evidenced-based therapy approaches (e.g. cognitivebehavioral therapy (CBT) and dialectical behavioral therapy (DBT)). For example the BlueIce app, offers a variety of functionalities including strategies based on CBT and DBT, mood diary, mindfulness exercises, and mood-lifting activities [27]. While there are advancements in the field of smartphone-based sensing in a range of mental health categories (e.g. [67, 69], the self-harm space remains relatively disconnected. In a systematic review of digital interventions for self-harm, there was a clear gap for mobile sensing solutions [73]. The majority 
of studies focused on offering evidenced-based therapies (e.g. acceptance-based therapy [65]. In the same review, only one study designed a game-like app called Therapeutic Evaluative Conditioning (TEC) to increase aversion to self-harm [25]. The results from 1 month trial of TEC showed reduction in self-cutting episodes by almost $40 \%$.

The aim of this research was to gain an understanding of young people's experiences of self-harm, and the triggers that might lead to a self-harm episode, in order to inform our understanding around potential approaches we might take towards predicting self-harm episodes using mobile interactions. To do this we first conducted interviews with a total of 4 mental health counsellors who had experience of working with young people who self-harm, to understand possible triggers for selfharm and the types of interventions that might be suggested in an attempt to prevent an episode. We then conducted an online survey with 37 young people who self-harm, to understand their attitudes towards the collection of social, location and app use data via embedded mobile sensors. Participants were asked about the data types that they would be happy for a mobile application to collect about their everyday lives, if it were to ultimately support them with their mental health. Finally, we conducted a workshop with 11 young people with experience of self-harm to further explore how a context-aware self-management application might be used to support them when they had the urge to self-harm.

Our work provides a vital first step towards understanding how mobile sensing might be sensitively used to predict and help prevent self-harm episodes. Our paper provides three contributions to HCI: 1) insights into how young people identify and manage triggers for self-harm; 2) considerations to sensitively create mobile sensing platforms that might be acceptable to young people; 3 ) design insights for future development of mobile applications to support young people who self-harm.

\section{BACKGROUND}

\section{Understanding Self-Harm}

Non-suicidal self-injury, commonly known as self-harm, refers to thoughts and behaviours relating to the deliberate and direct damage of body tissue without suicidal intent [44]. Selfharm behaviours include cutting, burning, scratching, and selfhitting [71], and can vary in terms of function, severity and trajectory [11]; many individuals report using several of these methods to injure themselves [24]. Thoughts related to selfharm are defined as a serious desire to engage in self-injury, usually happening when the person is alone and experiencing negative thoughts that last for 1-30 min; with occurrence of up to 5 times per week [46]. Though, by definition, self-harm occurs without suicidal intent, suicidal behaviour and self-harm can coexist [45] and acts of self-harm are a strong risk factor for subsequent suicide attempts $[62,72]$.

Young people who engage in self-harm do so for a multitude of reasons, both intrapersonal (e.g., low self-esteem, impulsivity, poor coping skills) and interpersonal (e.g., bullying, breakdown of a relationship, childhood trauma) $[19,58]$. According to the Experiential Avoidance Model (EAM), people engage in self-harm as an attempt to regulate and reduce overwhelming emotional states [17]. These unwanted emotional states can be triggered by stressful life events that the person perceives as particularly overwhelming and difficult to handle [44]. They can also be caused by underlying psychological difficulties, such as depression, anxiety, low self-esteem, relationship difficulties, and life stressors. Correlational and longitudinal research has identified multiple risk factors for engagement in self-harm including depression and anxiety, low self-esteem, childhood trauma, alexithymia (i.e., difficulty in identifying and describing own emotional states), impulsivity, and attention and conduct difficulties [14, 19, 57].

\section{Ecological Momentary Assessment (EMA) of Self-Harm}

Cross-sectional and longitudinal studies may help to understand long term risk factors of self-harm, but struggle to elucidate the more immediate contextual factors contributing to thoughts and behaviours around self-harm as and when they happen. Retrospective techniques (e.g., interview studies) that attempt to study these factors are prone to cognitive bias [54] and so more recent research has sought to use Ecological Momentary Assessment (EMA) techniques that gather real-time, real-world data on self-harm using mobile phone and web-based applications to encourage individuals who engage in self-harm to track their mood, situation and self-harm behaviours (e.g., [30, 54]).

EMA research has shown that self-harm engagement is preceded by an increase in negative emotions and followed by a decrease in these same emotions (e.g., [3, 6]), lending empirical support to theories that conceptualise self-harm as a form of emotional regulation [43] through a physical 'release' of pain. Importantly, this research has shown that the increase in negative emotions preceding self-harm engagement takes place hours before $[3,6]$, suggesting there may be a critical window wherein preventative measures may be employed.

Other research taking an EMA approach has also highlighted aspects of the situational context contributing to self-harm behaviour, such as being alone [46]. However, such research is limited. A recent review of EMA studies found that the majority of studies had focused on understanding the emotional context of self-harm behaviour, rather than cognitive or situational factors [54]. This may be because many participants struggle to articulate the motives of their self-harm behaviour, including cognitive and situational factors [3]. The same review also highlighted a lack of research using EMA with adolescent samples.

There may be particular merits to taking a technology-based EMA approach with young people - to understand the situational triggers of self-harm that are under-explored-given the central role technologies play in adolescents' lifestyles [10].

\section{Self-harm in Computer Science and $\mathbf{H C l}$}

Mental health and wellbeing is a growing application area for computing and human-computer interaction $[7,20,52,53$, 64]. Computing in mental health has primarily been leveraged for (1) explorations of online resource use (e.g., social media, 
online communities) by those who engage in self-harm behaviours, and (2) use of technology (particularly mobile apps) as an intervention to monitor or reduce self-harm behaviour.

\section{Studies of Online Behaviour}

Online resources have been identified as the preferred source for information and communication amongst adolescents who engage in self-harm $[60,21]$. Online resources can help to overcome problems of access and awareness [34] but are also perceived as being easier to engage with than face-to-face support, allowing young people to be more open about their behavior and feel less judged [33]. Thus, the Internet can provide anonymity, acceptance and support at a safe distance [38], allowing adolescents to overcome their fears surrounding shame and stigma to access important support and information. However, these same qualities that can make the web a safe space for supportive recovery communities also allow for the sharing of incorrect and deliberately harmful information [49].

Pater and Mynatt coined the term 'digital self-harm' [49] to describe online activities that contribute to self-inflicted intentional, non-suicidal harm. Their definition includes both direct (e.g., self-wounding) and indirect (e.g., eating disorders, reckless behavior) self-harm, and suggests directions for future research that incorporate a wide set of disciplinary and theoretical approaches. (Note that this same term has also been used in the literature to describe other non-physical harmful behaviors directed at the self [48]). However, for the purposes of this paper, we use the term self-harm to refer only to 'direct' harmful activities. Digital self-harm activities have emerged even in very general studies of adolescents' online behaviour [51] and in studies of other mental health conditions (e.g., depression [2], eating disorder [50]), however a small number of researchers have explicitly focused on online representations of self-harm $[13,22,26,23,31,36,40,41,56$, 70]. For a review of studies relating to social media use for the discussion of self-harm and viewing of associated content (1998-2014), see Dyson et al. [22].

Several studies of digital self-harm have focused on the use of hashtags to identify relevant content. Pater et al. [50] identified nineteen hashtags related to self-injury in their analysis of eating disorder content on Twitter, Instagram and Tumblr. These 19 tags have significant overlap with tags co-occurring (on Instagram) with \#depression in an analysis by Andalibi, Ozturk \& Forte [2]. One heavily-used tag from both datasets, \#sel fharmmm, was used by Moreno et al.'s [41] to establish a 'parlance' of self-harm that incorporated a set of otherwise ambiguous terms. Using Instagram to identify an initial set of tags that were often used alongside \#sel fharmmm, Moreno et al. resolved ambiguous tags by studying their use on other social media platforms and Google Images. In so-doing, they were able to triangulate and identify a set of relevant tags that might otherwise have been considered innocuous (e.g. \#blithe); of the eighteen tags determined to be self-harm related, only six triggered Instagram's in-built content advisory redirect service. Similar efforts to build a corpus of terms used in social media hashtags for (German) self-harm related content have also been made by Brown et al. [13].
Visual social media platforms have been an important area of concern in the study of digital self harm [2, 5, 13, 31, 36, 40, 41, 50, 56, 70]. Pater et al. [50] noted the presence of selfharm related images in their study of eating disorder content on Twitter, Instagram and Tumblr. Miguel et al. [40] conducted a manual review of items tagged with \#cutting and posted to Twitter, Tumblr and Instagram platforms over a period of six months. They found a high prevalence of graphic posts (i.e., cuts/scars $51 \%$, blood $17 \%$, or injury paraphernalia $22 \%$; total $60 \%$ ) and negative self-evaluations (46\%), particularly on Instagram. References to other mental health conditions were also very common (e.g., depression $78 \%$, eating disorder $43 \%$ ). A significant minority of posts were used for more positive purposes, actively discouraging self-harm (10\%) and providing or signposting recovery resources $(5 \%)$.

Other assessments of social media have focused on individual platforms. For example, studies of Instagram [13] and Tumblr content [56], posted in 2015 and 2016 respectively, verified the high prevalence of graphic wound depictions seen in prior studies. However, the latter study also noted the presence of content that did not directly depict self-harm (58\%) such as selfies and snippets from popular media - these indirect images were more likely to carry recovery-related messages and to be reblogged by other users [56]. Attempts to automate detection of self-harm content have also emerged; Wang et al. used machine learning to automatically identify self-harm related content on Flickr, finding clear patterns in language (tags, captions), social responses (likes, comments) and time of posting for self-harm content when compared to a control image pool [70]. Similar temporal and social patterns have also been seen in manual analyses [13], with content depicting severe wounds generating more comments than moderate and mild injuries. Manikonda et al.'s [36] study of Instagram posts also leveraged learning techniques, identifying self-harm as one of several mental-health disclosures made on the platform.

A small body of research has attempted to explicitly explore the role of social media use in real-world behaviours and clinical outcomes related to self-injury $[5,31,15]$. Jacob, Evans and Scourfield [31] conducted semi-structured interviews with twenty-one young people with a previous history of self-harm (sixteen had sought professional help for self-harm and eight had engaged with emergency healthcare as a result of selfharm). Individuals described how the internet reinforced their self-harm behaviours by embedding them in a community of peers for whom self-harm was as much a part of the daily routine as making a cup of tea, how digital communities allowed for sharing of previously unfamiliar techniques for self-harm, and how online shopping provided mechanisms to anonymously circumvent age-restrictions on razors and other blades. Interviewees highlighted the importance of online visual media in triggering an immediate desire to self harm, and its role in encouraging more extreme self-harm behaviours. Carey et al. [15] conducted surveys and interviews with twenty-nine young people as they engaged with emergency healthcare for issues suicidal- or other self-harmful-behaviour. Whilst some participants reported that they posted to social media because of its anonymity and presence of like-minded individuals, others reported the presence of family members and peers as a 
motivation for avoiding posting personal content on the platforms - some participants explicitly referred to the creation of additional social media profiles to avoid this issue. Arendt et al. conducted a two-wave panel survey demonstrating that exposure to self-harm content on Instagram was associated with self-harm and negative emotional well-being, and also predicted self-harm and suicide-related outcomes one month later [5]. Our own interviews with adolescents target individuals who have a self-defined prior experience with self-harm but consider online behaviours as just one aspect of a broader technology ecosystem. Further, our interviews deliberately prompted young people to consider the role of technology in managing self-harm, a topic not explored in these studies.

\section{Technology Interventions}

George [26] noted that the logical next step for technology is the development of systems to detect vulnerable adults and target interventions. However, there is currently a very broad body of knowledge to inform these future interventions. Birbeck et al. conducted their 'Self-Harmony' hackathon inspired by value sensitive design approaches to encourage seven mixed-expertise teams to ideate in the space of technology for self-harm [9]. The two-day event encouraged the production of designs intended to (1) aid emotional coping, (2) raise awareness of self-harm, and (3) explore harm reduction. The resulting prototype designs included two mobile apps, three web-based services, and two physical artefacts; the designs were critiqued by expert stakeholders, with the majority of attention being spent on the two physical artefacts and one mobile app. As a result of their experiences, Birbeck et al. identified several key challenges for the development of digital technologies for self-harm. Firstly, that there is a need to avoid burdening friends and family for emotional support - such parties may, in fact, need their own interventions. Secondly, that technology should consider the complexity and changing nature of individual's moods and behavior. Thirdly, that health and care stakeholders may have conflicting priorities. Finally, that although conventional hackathons are not well-suited to creating lasting technologies that address real-world problems for human users, follow-on engagement can extend involvement to allow for far-reaching and impactful technologies.

Research has also led to the creation of longer-lived technology interventions targeted at self-harm behaviors. Hetrick et al. [28, 63] used a studio design methodology, and codesign workshops with both young people and clinicians, to design a mobile app. Core functionality included mood monitoring, access to timely support responses, and embedded interventions (both distractions and personalized mood lifters). Outside of the research setting, Calm Harm [59] is a UK-developed application produced by a teenage mental health charity. Based on a Dialectical Behavioural Therapy (DBT), the app supports users in six tasks designed to be carried out whenever users feel the urge to self-harm.

\section{OUR STUDY}

\section{Ethics}

The research presented in this paper received ethical approval from the Lancaster University research ethics committee. All participants gave informed consent to take part in the study.
Survey participants were presented with the study information on the welcome page of our online survey and were free to abort the survey at any time. All workshop and interview participants provided written informed consent. Two social workers, who helped with organising the workshop, were also present to help with any discomfort caused from the discussions in the workshop.

\section{Recruitment}

Interviews were conducted with two pairs of counsellors whose clients had engaged in self-harm activities, and a focus group with eleven young people aged 18-25 who selfidentified as "having self-harmed in the past and are not in a current crisis or suffering from current severe mental health or other high risk behaviours such as drug and alcohol misuse". Counsellors were recruited through an existing online support platform. Similarly, young people engaging in face-to-face activities were recruited through a charitable organisation that provided support for mental health and emotional wellbeing.

The online survey targeted those aged 16-25 who again selfidentified as having experience with self-harm and could answer about themselves or about someone they know who has been affected by self-harm. Participants were recruited from the same platform as the counsellors.

\section{Interviews with counsellors}

We conducted two paired interviews with counsellors $(n=4)$ over Skype to gain a deeper understanding of a typical young person who self-harm: their recovery plan, the main triggers young people frequently report, the techniques counsellors use to identify these triggers, and finally the treatment approaches including the types of interventions they find most useful.

\section{Supported Interview Discussion}

Counsellors were encouraged to fill in a persona worksheet prior to attending the interview, thinking about a typical young person who self-harms. During the interview, counsellors discussed the similarities and differences between personas. We then carried out group discussions scoping out triggers. We wanted to identify the most reported triggers; the benefit of identifying triggers with individuals during a counselling session; how counsellors help young people identify their triggers; what information helps the counsellors to identify triggers; and once triggers are identified how young people are supported to manage a self-harm episode. Finally, we explored treatment approaches by discussing current professional practice; the resources and material used in treatment; reflections on remote counselling methods and resources used; and current gaps in remote counselling.

\section{Survey}

Following our interviews we had an understanding around some of the major triggers for self-harm and how these might be mitigated or supported through different distraction techniques. We next wanted to understand how smartphone technology might be best utilised in order to deliver context aware interventions. In order to do this, we first wanted to understand young people's perceptions of mobile data collection, and how appropriate certain techniques might be. 
We conducted a survey, hosted on an online counselling platform for a total of 3 weeks. The survey encompassed 19 questions surrounding mobile sensing approaches which have the potential to collect data on user's physical and social interactions. It focused on the type of data young people would be happy to give access through an app. For instance collecting data on the types of apps they use; time and duration of app usage; what messages they are sending through their phones; what health related websites or apps they use; location data; information about other people's phones nearby. In addition we wanted to know whether young people would access an app when feeling distressed; would they be interested to have a phone "companion"; and how would they like this "companion" to help them in distressing times; whether they currently use their phone when feeling distressed; if yes, how and what kind of apps they use. Survey responses were collected via simple questions responses (e.g. would you be willing to let an app collect information about when you are using your phone: yes, no, I don't know) and free-text input.

\section{Workshop with Young People}

Finally, we recruited 11 young people to take part in a workshop to better understand the context in which they experience self-harm: their coping mechanisms, support resources, online presence in terms of self-harm, views on the role of technology for managing self-harm, and perceptions on data privacy and trust. The workshop was held at the charitable organisation venue familiar to the participants. While we did not intend to recruit participants from only the LGBTQ+ community, all participants (except 1) knew each-other, mostly through the LGBTQ+ group. However we did not ask participants to disclose or discuss their sexuality (as this was not the focus of our research). Each participant disclosed their gender identity at the beginning of the workshop by discussing their preferred pronouns (male $n=6$, with only $n=2$ cisgender males).

Participants received $£ 20$ Amazon voucher for their time. The workshop lasted approximately 2 hours, and was audio recorded and transcribed with the participants' knowledge and consent for qualitative analysis.

\section{Workshop Activities}

Due to the potential sensitive nature of the topic area (i.e., self-harm), we asked participants to work with personas, in order to allow for a level of disassociation from their own personal experiences [16]. For the first activity, using a provided storyboard sheet, participants each created a persona that, to them, represented a person with lived experience of self-harm. They were prompted to think about their personas in terms of age, gender, brief overview of self-harm, severity, what makes that persona feel happy/sad. This was followed by sharing created personas through the group discussion and discussing similarities and differences between personas.

We then split the group into three and asked each group to select a persona to work with for the next activity ("A day in the life of..."), which aimed to better understand a young person's activities and interactions during a 24-hour timeframe. Participants were provided with a timeline and were asked to think of a typical day for them, identifying specific activities

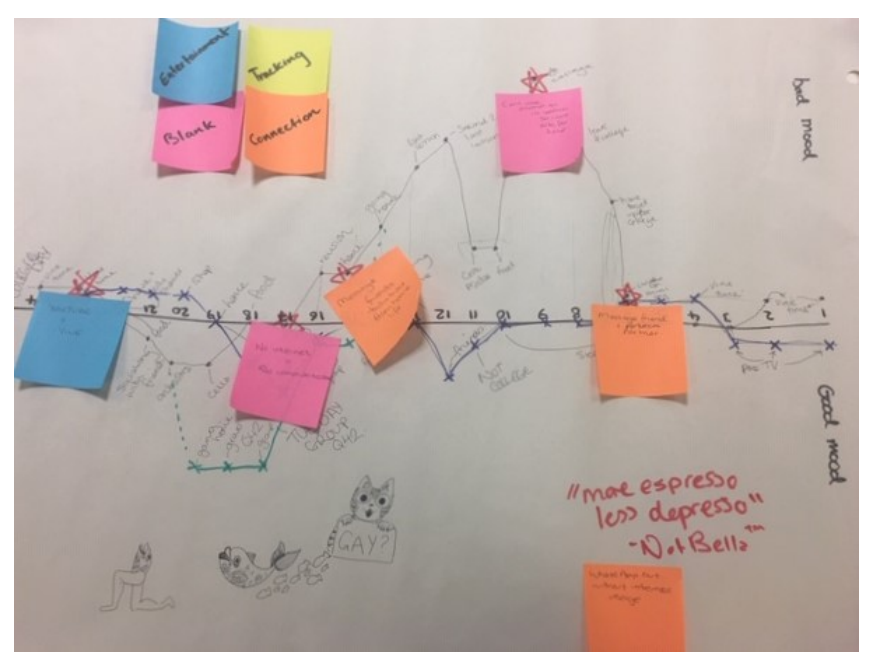

Figure 1. "A day in the life of ..." activity sheet.

they might do, and how they felt while doing them. Emotion was represented on the y-axis of the timeline (see Figure 1). Participants were then asked to think about what triggered their lower moods and identify any instances when they felt that they might be likely to self-harm. They were then encouraged to discuss the strategies they use for managing these low moods. The young people modified the activity during the workshop, to differentiate between the mood in weekdays and weekends.

Following this, the final activity involved exploring different types of apps and how they might be used to help in the management of self-harm. Three sets of differently coloured 'category' cards were provided, on which participants could write their responses: 1) Entertainment (e.g., games, video platforms), 2) Tracking (e.g., step counter, food diary), 3) Connection (e.g., social media, SMS). Blank cards were also provided if participants had other types of applications they wished to discuss. We asked them to think about how each category could be used during low moods reported in the previous activity. We asked each group of participants to choose the top three features they liked most from the apps they had identified. A group discussion then focused on refining insights around what young people might want in an application to directly support them with self-harm, and the type of data that might be required to develop such an application, if applicable (e.g., automated prompts to carry out exercise when sedentary would require access to their phone's accelerometer).

\section{Data Analysis}

Two members of the research team followed an iterative process of conducting an inductive thematic analysis as outlined by Braun and Clarke [12]. This process started by familiarising with the data, generating initial codes, clustering and gathering data into potential themes, reviewing the generated themes against the data, producing defined themes, discussing any discrepancies between coders before agreeing on a final set of themes. Survey data underwent a content analysis [29], which involved looking for emergent themes relating to each specific question. 


\section{FINDINGS}

\section{Interviews with Counsellors Findings}

A total of four overarching themes were generated from our thematic analysis: (1) Understanding Triggers; (2) Distraction Tools; (3) Reconnecting with the Body; and (4) Tools to Support Identification of Emotions.

\section{Understanding Triggers}

Counsellors highlighted the importance of helping young people who self-harm to identify their individual trigger points. In particular it was noted that monitoring when self-harm was occurring was a useful starting point for understanding risk: "you find out what times of day they find self-harm to be worst, I tend to find that's at night time with most young people, when they're on their own." (C2). One resource they used widely to support this discussion was tracking of mood or feelings: "Some people really need that, very simply just smileys from one to ten and maybe good things today and bad things today" (C3). C1 highlighted the importance of tracking both good and bad feelings and not just focusing on the problems: "I'll get them to record times when they felt distressed or really upset. I'll also get them to record a time or a situation when they felt really good". This approach was perceived to be effective in helping young people understand that fluctuations in mood is natural: "we're able to say that there's a continuum if you like throughout the day and we can go up and down [...] that's $O K$ " $(\mathrm{C} 1) . \mathrm{C} 1$ discussed how they would facilitate a thinking space to reflect on three things: "how they felt, what they were thinking and what they did" (C1), used as a way to become aware of triggers i.e., people, environments, or situations. This reflection was carried out through a "talk me through your typical day" activity where they would talk about a situation that led into self-harming: "almost do a mini timeline of what might have happened so that they can start to recognize it [trigger point]" $(\mathrm{C} 1)$.

\section{Distraction Tools}

Distraction techniques were discussed broadly as one of the most effective methods for providing "head space" to the young person with self-harm urges, to refocus and gain back control over their actions. C2 described a client who had started to draw when they felt like self-harming: "they didn't even feel like they were concentrating but when they looked at what they'd drawn when they were feeling really angry and overwhelmed... they felt that actually afterwards they didn't have the guilt of 'I've just cut myself again'... they actually felt quite good about it", whereas C1 discussed the value of writing: "whenever you write it down. . it almost is a chance to refocus again so you're detaching, you're keeping that at that healthy distance". C3 described regularly recommending a specific mobile application (Calm Harm [59]) to their clients, which was seen to allow them to take time to refocus their attention: "it has things like a timer where when you have the urge to self-harm you can put a timer on to say 'OK I'm not going to self-harm for ten minutes and then I come back and see how I feel now"' (C3). While all counsellors recommended distraction techniques as useful tools in managing self-harm, C3 highlighted the importance of working with clients to understand their own behaviors and develop appropriate coping strategies that work for them: "... someone who just wants to feel something, that's why they self-harm, it makes no sense to suggest to them to take a bath... that's not going to do what self-harm does for them".

\section{Reconnecting with the Body}

There was a strong emphasis on desensitizing self-harm through the provision of psycho-educational tools. C1 recommended using an infographic to help young people understand what is going on in their body during anxiety and panic: "it's [a] figure of the body and... a short synopsis of what the threat response is... an arrow with all the different physical activations that are happening, so that they can understand that these are normal responses... when you don't know what's going on you think you're dying". There were discussions around the importance of reassuring young people that the physical sensations they feel are natural but also providing them with accurate information about their body. C2 explained that when someone experiences an increased heart rate, they may automatically assume they are having a panic attack: "especially the younger ones, it makes them panic even more", which could then trigger the young person to self-harm as a way to deal with the panic. At that point, this visual image of what is happening to their body was seen as an effective technique to help young people understand that this physiological change is a natural body's response: "this is what's going on, this is very natural, this will start to drop, and they have that visual image of what's happening in their body" (C1). This knowledge was perceived to empower young people in gaining back a sense of control over their body: " $a$ lot of people who self-harm feel like they're completely out of control, they can't stop it from happening. It's reminding them that actually they are in control of themselves, you know, self-harm isn't controlling them" (C2).

\section{Tools to Support Identification of Emotions}

The counsellors emphasized that young people need to learn how to handle feelings and emotions, which can help them build resilience to daily challenges. This was seen to be particularly challenging: "it's finding words for what's going on inside, it's expressing it" (C3). C4 discussed how counsellors would guide young people towards developing a language with which to express their emotions "what could it be?, am I angry?, am I lonely?, am I pissed off ... what else can I do apart from self-harm" (C4). Issues related to expressing feelings were raised frequently by the counsellors, and it is therefore necessary to provide more creative ways to enable this communication: "I would use a lot of quotes for young people, [like] "you're not for everyone and that's $O K$ ” and there's another poem that I would send sometimes, maybe say they've come through a really difficult time..., its' that kind of metaphor, almost like a story" (C1). Once emotions could be identified the counsellors discussed a need to self-quantify the level of emotion in order to determine the best coping strategies to employ: "listening to music sometimes doesn't help anymore because the tension is too high. So we discussed to maybe have a really cold shower when the tension is proper high, when it's medium to listen to music and scream, and when the tension is low to go for a walk" (C4). 


\section{Survey Findings}

A total of 37 people responded to our online survey (mean age 16.8 years, sd. 2.1). The sample was predominantly female (21 female, 6 male, and two people identified as gender-fluid).

The majority of respondents said that they would be interested in an app to support self-harm $(\mathrm{n}=31)$ and were happy to share their phone usage time $(n=24)$, location $(n=22)$, and what health websites or apps they use $(n=22)$. Over half of participants $(n=22)$ reported that they use their phones to help them when they were feeling distressed. We used an open question to further probe how apps were being currently used, identifying three major coping strategies: consuming audiovisual media $(\mathrm{n}=8)$, e.g., "Use to look at things that I love or even just to watch funny videos when I am in a bad way" (P9); seeking peer-support $(\mathrm{n}=8)$, e.g., "I try and text my friends to try and distract myself" (P11); and using meditation or mental-health apps $(n=11)$. For one participant, the phone itself acted as a barrier, protecting them from engagement with other people at times of distress: "It's a social crutch and helps me to cope in a panic attack. If I'm on my phone, people tend not to bother me" (P35).

Further open questions asked respondents to discuss aspects that they wished their phone would provide to help them during times of distress. We identified several themes relating to desired support. The first was around helping the user regain a sense of control by calming themselves down during distress $(\mathrm{n}=10):$ "Help me to calm and see things rationally" (P17); a desire for human connection and communication was also discussed (n=5): "Be able to bring up immediate help lines that it will call automatically for you. Sometimes pressing that call button is the hardest moment you'll ever face in getting help" (P5); "Allow me to talk to someone who I don't know who will understand what I'm going through" (P14). Gaining advice through an application was also seen to have value $(\mathrm{n}=5)$ : "Give helpful suggestions with how to deal with situations" (P23); "It would provide virtual support when you have got really low, and reminders that it will not stay" (P24), as well as app nudges that would remind the user that they are not alone (n=9): "Random checks to ask how I am or what I'm up to. Just random questions even when I'm not using the app so it always reminds me there's something to help" (P29).

Finally, we identified two high level coping strategies that people wished for a hypothetical phone companion to support them with. Participants discussed opportunities for the phone to initiate distraction techniques (n=9) "Shut off social media, automated playlist? (like linked to Spotify), provide distractions eg minigames/activities" (P1); "Something that will just talk to me and try and distract me from my urges" (P11) and providing CBT techniques to help the individual think clearly and rationally about their thoughts and emotions $(\mathrm{n}=5)$ : "Talk to me about the reasons I'm distressed. Remind me of my goals and my self-worth. Comfort me even in the irrational moments and get help if it was seriously concerned that it would not be able to stop me from committing a harmful act” (P5).

\section{Workshop with Young People Findings}

A total of four overarching themes were generated from our thematic analysis: (1) Understanding Mood Changes; (2) En- tertainment as Distraction; (3) Tracking Ambivalence; and (4) Social Connection.

\section{Understanding Mood Changes}

When sharing their personas, young people repeatedly highlighted lack of sleep, loneliness, overthinking, societal pressures, gender identity, crowded environments, academic pressure and tiredness as the main factors affecting their moods. The most distinctive factor leading to a peak in mood was socialising: "we have break at quarter past ten which is always quite nice, so I'd say [my mood] goes up a little bit more, because I go and see other people" ( $\mathrm{P} 4)$, whereas tiredness and lack of sleep was seen as leading to a dip in mood: "Because I'm starting to feel a bit tired so I'll start to feel a bit like hmm, like as much as I love socialising, sometimes I just get to point where I kind of want to stop now" (P4). There was much distinction between mood during the weekends and weekdays. In general, young people raised less concerns related to stressors during the weekends: "Things are good, life's good, there's not too much stress around, apart from perhaps with the revision, but even with the revision you're in a good place" (P4). Whereas weekdays, particularly when attending school, were linked with excess stress and therefore lower mood: "At college you realize you're stuck, and if you have no friends it's sad. Going home, so you have to deal with family and sometimes family can be viscous, and then you've got revision, which just makes you worry more about college and basically just a continuous cycle of worry" (P1). Night time was also repeatedly linked with self-harming: "cos it's night time, you're alone with your thoughts and no distractions and there's all that going on" (P7).

\section{Entertainment as Distraction}

Young people shared that they widely use media entertainment as a distraction technique. Mostly listening to music as a calming solution; watching vines, or YouTube videos; playing mini games like Snake and Tetras; and drawing. These suggestions confirmed the distraction techniques recommended by the counsellors previously. In discussing mobile apps as tools for offering therapeutic support for self-harm, young people described mobile phones as "the first thing I reach for in any situation" (P3) and acknowledged that "it's the only thing that will connect you to things that might still help you" (P5). They also found it to offer a confidential/ personal space: "no one goes near my phone, not unless they want to lose a hand, I won't let anyone near" (P1).

The young people discussed further how they thought apps could help in distracting from self-harm. Firstly, they envisioned offering activities through the app that require less concentration: "you don't have to think about it" (P5), as this was seen: "like absent minded doing something else" (P3). For example, doodling was favoured as it does not require much thought: "I don't know, just like really simple ones that anyone can do. Draw a heart, colour this in, colour by numbers is always fun" (P7). Whereas activities requiring concentration were dismissed: "no puzzle games because if they can't solve the puzzle they're going to get even worse" (P9). They even suggested offering pre-written messages for send- 
ing to friends: “cos if you're like panicking you don't want to be trying to focus on writing something out" (P1).

Secondly, they liked to engage in activities in more than one way, for example, listening to music and drawing at the same time: "It's distracting and it's like calming, especially if it's something you can do whilst still listening [to music]" (P4).

Thirdly, they favoured the app to provide instructions about the activity, for example instructing the user to draw a specific image: “And if it's telling you what to do as well, it's kind of like taking control, it's like you're taking control 'cos you're following the instructions" (P3). This was seen beneficial for two reasons, first to follow instructions when distressed without requiring much thinking from the user, second, to be able to rebel against the app by not following the instruction, which was perceived as a way to regain a sense of control: "And if you want to, you don't have to follow the instructions, then it's rebelling which also helps" (P3). Lastly, participants explained how light-hearted content could help to distract them: "I'm one of those people who loves this, extremely useless facts, that are like did you know that this blah blah blah" (P7). These "useless facts" were seen as "something really stupid to counteract with something that's very serious" (P1), which made them feel better. In this sense, they asked for very random and unusual facts to replace the thought of self-harm: "stupid so that they remember it and it sticks in your head instead of something else [self-harm]" (P8).

\section{Tracking Ambivalence}

When prompted about tracking, the immediate reaction was: "oh I don't like it". However, when young people started expanding on their tracking experiences, it was evident that they disliked the action of tracking and labor-intensive act of inputting data: "I tried to do it before and I just didn't really keep up with it" (P4), as opposed to enabling passive monitoring.

In addition, young people associated tracking activities such as diaries, with reflecting on self-harming events: "I think the main problem with tracking is like it makes you think about it" (P1). Not only this was associated with causing further distress: "It's always nicer to feel like oh it's been three months, than oh I've been thinking about it this whole time and keeping track of every single day, then it just gets stressful" (P3), but also tracking the negative was perceived to give rise to negative thoughts: "and then if you think about it you're more likely to do something [self-harm]" (P4). The young people raised concerns about the type of feedback a tracking app may provide: “It's the crippling realisation of how shit your life is" (P11) and "Cos you're looking at it thinking well I just feel like crap all the time” (P4). They preferred not to see their mood explained or visualized: "You don't really want to see how anxious you're feeling” (P1), instead they favored the app to provide tips or to suggest activities when recognized a low mood: "so if you're feeling like this, would you like some tips, have a draw, do some drawing. Now and again it just pops up with like a tip, that would be quite good" (P3), or compliment and affirm when things are going good: "Or a nice little compliment" (P2).
Furthering this, the participants found compliments for positive actions e.g., exercise or completing a task "rewarding" and considered it to improve mood in other contexts e.g., school: "if you're actually walking and every so many steps you get a free compliment, then when you get to school you could just open it and be like I did well for coming to school, oh my God I think I'm going to pass my exam" (P3). It seemed that receiving affirmations throughout the day, could really help young people to get through the task they normally struggle with: "Maybe if you told the app where your college was or whatever and when you got there it was like you got it through" (P3) or "A little light at the end of each lesson like good job, you got through physics” (P1).

Overall, they did not mind if the app prompted easy to answer questions about their moods, but wanted to ensure that this self-report would be very simple: "It's like an easy question to answer, it doesn't feel so bad to have tracking in it, but if it's actually in-depth questions then you wouldn't want to answer those" (P7) and were happy to answer general "how you're feeling?" questions every now and then through the app. This also led into strong suggestions for sending reminders through the app, with examples including: "drink water", "eat something”, "take your medication”, "stop slouching”, "stretch out", "you've been up for five hours, it's probably time for you to sleep", and "if you've not moved from the same spot in like three days, maybe just go on a bit of a walkabout”.

\section{Social Connection}

Participants valued social media for two reasons. First, for enabling better connectivity with friends: "that [social media] helps me 'cos it's like you're in constant contact with friends, you're less likely to be down" (P1). Second, for offering lighthearted distracting content like aesthetic pictures and mood boards: "look at animal pages on your Facebook, look at this ferret, look at this cat" (P3); "Oh my God I would kill for an app that just sends me like cute pictures of hamsters or cats every now and then, like are you in a bad mood? Have a cat, and then suddenly everything is solved" (P4).

When asked about how they would feel if the app is collecting data in the background about the amount of times they spent on social media, young people raised concerns about the accuracy of this data: "I think you'd have to find a way to make sure it's distinguished between like running in the background and like actually being on it" (P6). However, they felt that the app could use this data, if collected accurately, in a positive way: "if you've been on Facebook for like six hours straight, can you please remember to eat and drink. 'Cos I've done that, I have gone an entire day sat on my laptop and just forgotten to eat" (P1). They suggested daily obliteration of data rather than storing raw content within the app: "I think ideally it should collect it and then delete it at the end of the day "cos it should be like a daily thing of like how are you doing today" (P3). We then explained that this data can be used to improve the algorithms in a way to offer enhanced support and suggestions to the individual. Once the use of data was explained, they felt better about allowing the app to have access to such data: "yeah that makes sense" (3), and "yeah that works" (P1), but they wanted to know/ control which apps and what content 
they are allowing access to: "You should put an option where it shows which apps it's allowed to access" (P2).

\section{DISCUSSION}

We have described a series of consultation activities with young people with experiences of self-harm (surveys and workshops) and counsellors directly supporting this community (interviews), to explore how smartphone-based technology can be used to better understand, prevent and predict self harm. Though we analysed each of our data sets separately, there was substantial overlap in the themes that were developed reflecting consensus among young people and counsellors as to the potential role of smartphones. In the following discussion, we reflect on these findings to develop a set of design considerations that will aid the future development of smartphone-based interventions to support young people with experiences of self-harm.

\section{Understanding Self-Harm}

Young people and counsellors reflected on their use of selftracking methods (e.g., mood diaries) to identify multiple factors related to mood fluctuations and self-harm, including tiredness, social isolation, crowded environments, and academic pressures - supporting past research with similar findings (e.g., [68, 47]). While the findings suggest common triggers, the combination or sequence of these triggers, as well as the exact consequences for mood, were positioned as being unique to individuals. As such, both young people and counsellors emphasised the importance of understanding the mood dysregulation and triggers associated with self-harm on an individual case-by-case basis. These findings echo those of Berrouiguet et al. [8], who highlight the importance of taking an individual approach to treating mental health issues due to the diversity of triggers that might be seen.

Both young people and counsellors identified a range of contextual factors that contribute to self-harm behaviour, including times and places. For example, acknowledging the difference in mood fluctuations between weekdays and weekends and the reasons underpinning this (e.g., lack of friends at school), or acknowledging their increased urge to self-harm at night-time when they are more likely to be alone. By acknowledging and identifying specific times and places that triggers occur (e.g., through location sensing, tracking mood at different times of the day, as defined by Torous et al. as "momentby-moment quantification of disease phenotypes" [66]) we can build a better contextual understanding of self-harm risk. Future HCI work has an important role to play in this, given that existing methods of understanding self-harm (e.g., mood diaries and qualitative studies) my struggle to accurately measure these contextual factors.

Importantly, future work should consider a multi-sensor approach to understanding triggers of self-harm among young people. Based on our findings, we suggest the most useful sensors would include those that collect information around time, location and social media/ entertainment platform usage. By integrating diverse smartphone-based data streams with smartphone-based EMA, HCI researchers can enhance the validity of trigger assessment for each individual, as seen in monitoring schizophrenia symptoms [69]. For example, we found that majority of participants thought that socialising has positive impact on their mood and so monitoring social cues through smartphone sensors (e.g., location, number of people nearby, time spent engaging in messaging activities through the smart phone) may be a fruitful means of establishing a more holistic picture of the situational triggers of one's mood. Coupling this mobile sensor data with mood self-report data, would help to gain a more detailed insight into users' $\mathrm{mood} / \mathrm{self}$-harm triggers, which can then be used to inform targeted interventions.

However, this type of data collection (i.e., using multiple sensors to collect comprehensive and sensitive information) raises important ethical issues [4]. Smartphone-based EMA may be viewed intrusive and self-reporting mood in situ has potential to be triggering. Participatory approaches that involve youth in the development of these technologies are important in ensuring the needs, wants and desires of vulnerable users are met. In particular, the nature of self-report items (e.g., wording and frequency of mood-related questions) would need careful consideration to ensure that young people are comfortable with their use. It is also important that users understand what they are consenting to, for which Areán et al. suggest to use consent quizzes prior to downloading an app [4].

\section{Predicting Self-Harm}

Next, our findings have implications for those interested in the prediction of self-harm using smartphone-based technologies. Both young people and counsellors reflected on how smartphones could be used to identify patterns of emotions and behaviours associated with self-harm. In workshops, young people expressed that they would be generally be comfortable with the idea of behavioural tracking through smartphonebased sensing, and would give access to their relevant personal data for this purpose. Survey respondents also expressed a similar level of comfort with giving access to their data i.e. phone usage time, location, and the type of health website and apps they access. Young people wanted the self-tracking through apps to be as automated as possible; they preferred a passive relationship with the app preferably tracking their behaviours in the background. This echoes findings by [1] who developed a smartphone-based sensing to automatically detect social rhythms in bipolar disorder to address the challenges associated with manual tracking [37].

However, understandably, not all participants were willing to share their data. Some young people felt apprehensive in relation to discussions of data for two reasons. First, they questioned the accuracy of collected data i.e. not carrying their phone at all times. Second, they initially did not want data to be stored about them, suggesting data should be obliterated on a daily basis. This indicates that, in future work aiming to engage large numbers of participants in such a sensitive topic area, we would need to take extreme care to ensure that any concerns about data capture and use were alleviated. This leaves us with two alternatives for future deployments; allow users to opt-out of particular data streams or further investigate people's concerns and inform them better. In previous healthcare monitoring research projects, participants were mo- 
tivated to share location-based data by the perceived benefit (economic or altruistic e.g. helping others suffering with the same condition [18]) and have reported being confident that it will be collected, transmitted, stored, and analysed following research guidelines agreed beforehand [61]. Additionally, the young people in our workshops were more willing to consider different types of data sharing once they knew more about the purpose of the data collection.

Future work should investigate how to incorporate machine learning approaches to develop multi-sensor prediction models that relate to self-harm triggers. To do this, we need to use participatory approaches to develop an understanding of the types of sensor data that people who self-harm might be willing to share in the development of context-aware tools and also to provide insight into the what these tools should look like. It is important to ensure the capture and translation of this data is conducted in a way that elicits trust from users [35], and so protection of privacy and trust development must lie at the core of any design work within this space. Safeguarding against any possible unintended consequences that might arise when relying on predictive tools is also important. Issues exist around the generalisability of predictive algorithm, which raises concerns about the responsibility of the app when over/ under predicting occurs. Future work needs to be mindful of these possible challenges and investigate how algorithms could be improved to minimise this risk (e.g., capturing and utilising consistent user feedback).

\section{Preventing Self-Harm}

Lastly, our findings have implications for the design of smartphone technologies that prevent self-harm. Our findings show that many young people already use their smartphone to manage their mood and self-harming. Young people described using smartphones to access audio-visual media, reach out for peer-support, access meditation tools, listen to music, watch videos online, play games, engage with others via social media and/or doodle, in order to alleviate the negative emotional states associated with self-harm. Counsellors described how many of these uses had been recommended during therapy or were consistent with therapist-recommended approaches. For example, counsellors described how they advocated distraction techniques in the form of drawing, writing, or taking time to refocus attention.

There are some mobile apps currently available on the market that advocate these techniques. For example, Calm Harm [59] offers six different types of intervention in response to self-identification of thoughts and feelings associated with self harm engagement, including "comfort, distraction, express yourself, release, random and breathe". The app is based on the principles of DBT, a widely-used and empirically-tested therapeutic technique for self-harm management [39]. For example, techniques provided in the comfort section include advice for controlling self-harm desires, which young people in our workshop identified as being important to them.

Our findings have important implications for HCI researchers concerned with the design of future apps aimed at preventing self-harm. First, interventions could be personalised to incorporate adolescents' individual preferences. Given that participants reported requiring different modes of support in different circumstances, app-based intevention should not only incorporate a variety of techniques (e.g., distraction, relaxation, human connection, advice, support, and cognitive behavioural techniques) but also possess the ability to be personalised in order to meet individual's needs. Second interventions could be automated through mobile-sensing of self-harm triggers. That is, mobile-sensing techniques described in the predicting section could be augmented with personalised interventions to maximise efficiency. Thus adolescents could receive personalised in-situ context aware interventions at the time where they need it most. Lastly, our findings highlight the importance of engaging both young people and therapists in the design process of app-based intervention. The extent to which commercially available apps have involved young people in co-design activities is unclear.

\section{LIMITATIONS}

Our recruitment method introduced a sampling bias. We did not intend to focus our recruitment on LGBTQ+ young people, yet most of our participants were members of the LGBTQ+ group within the counselling service that we recruited through. Since this selection was not within the aims and focus of this paper, we did not explicitly explore how sexuality intersects with self-harm. We acknowledge that sexuality, and the complexity that sexuality adds to the self-harm experience, are beyond the scope of this initial research. However moving forwards, we advocate for a more inter-sectional approach to examining self-harm in youth that accounts for factors such as sexuality, gender and ethnicity.

\section{CONCLUSIONS}

This study aimed to understand how smartphones can be used to better understand and support young people who engage in self-harm. Through our extensive empirical programme, we engaged with both young people who self-harm (using surveys and workshops), and their counsellors (using interviews). Our findings have yielded important insights into how designers can develop smartphone-based technologies in order to understand, predict and prevent self-harm. In particular, our findings highlight the potential for the development of in-situ, context-aware interventions facilitated by smart-phones that can support young people who self-harm when they need it most. They also highlight the importance of involving young people who self-harm, as experts through experience, and other important stakeholders throughout the design process.

\section{ACKNOWLEDGMENTS}

The authors thank all study participants for participating in this study. In particular, 42nd Street for supporting participant recruitment and hosting the workshops. This research was funded by the EPSRC-NIHR NewMind Network Plus.

\section{REFERENCES}

[1] Saeed Abdullah, Mark Matthews, Ellen Frank, Gavin Doherty, Geri Gay, and Tanzeem Choudhury. 2016. Automatic detection of social rhythms in bipolar disorder. Journal of the American Medical Informatics Association 23, 3 (2016), 538-543. 
[2] Nazanin Andalibi, Pinar Ozturk, and Andrea Forte. 2017. Sensitive Self-disclosures, Responses, and Social Support on Instagram: The Case of \#Depression. In Proceedings of the 2017 ACM Conference on Computer Supported Cooperative Work and Social Computing (CSCW'17). ACM, New York, NY, USA, 1485-1500. DOI : http://dx.doi.org/10.1145/2998181.2998243

[3] Holly E Andrewes, Carol Hulbert, Susan M Cotton, Jennifer Betts, and Andrew M Chanen. 2017. Ecological momentary assessment of nonsuicidal self-injury in youth with borderline personality disorder. Personality Disorders: Theory, Research, and Treatment 8, 4 (2017), 357.

[4] Patricia A Areán, Kien Hoa Ly, and Gerhard Andersson. 2016. Mobile technology for mental health assessment. Dialogues in clinical neuroscience 18, 2 (2016), 163.

[5] Florian Arendt, Sebastian Scherr, and Daniel Romer. 2019. Effects of exposure to self-harm on social media: Evidence from a two-wave panel study among young adults. New Media \& Society (2019), 1461444819850106.

[6] Michael F Armey, Janis H Crowther, and Ivan W Miller. 2011. Changes in ecological momentary assessment reported affect associated with episodes of nonsuicidal self-injury. Behavior Therapy 42, 4 (2011), 579-588.

[7] Bert Arnrich, Venet Osmani, and Jakob Bardram. 2013. Mental health and the impact of ubiquitous technologies. Personal and Ubiquitous Computing 17, 2 (2013), 211-213.

[8] Sofian Berrouiguet, Mercedes M Perez-Rodriguez, Mark Larsen, Enrique Baca-García, Philippe Courtet, and Maria Oquendo. 2018. From eHealth to iHealth: transition to participatory and personalized medicine in mental health. Journal of medical Internet research 20, 1 (2018), e2.

[9] Nataly Birbeck, Shaun Lawson, Kellie Morrissey, Tim Rapley, and Patrick Olivier. 2017. Self Harmony: Rethinking Hackathons to Design and Critique Digital Technologies for Those Affected by Self-Harm. In Proceedings of the 2017 CHI Conference on Human Factors in Computing Systems (CHI'17). ACM, New York, NY, USA, 146-157. DOI : http://dx.doi.org/10.1145/3025453.3025931

[10] Danah Boyd. 2014. It's complicated: The social lives of networked teens. Yale University Press.

[11] Katherine L Bracken-Minor, Meghan E McDevitt-Murphy, and Gilbert R Parra. 2012. Profiles of non-suicidal self-injurers and associated patterns of alcohol use. Journal of Psychopathology and Behavioral Assessment 34, 4 (2012), 552-563.

[12] Virginia Braun and Victoria Clarke. 2006. Using thematic analysis in psychology. Qualitative research in psychology 3, 2 (2006), 77-101.

[13] Rebecca C Brown, T Fischer, AD Goldwich, Frieder Keller, Robert Young, and Paul L Plener. 2018. \#cutting:
Non-suicidal self-injury (NSSI) on Instagram. Psychological medicine 48, 2 (2018), 337-346.

[14] Romuald Brunner, Michael Kaess, Peter Parzer, Gloria Fischer, Vladimir Carli, Christina W Hoven, Camilla Wasserman, Marco Sarchiapone, Franz Resch, Alan Apter, and others. 2014. Life-time prevalence and psychosocial correlates of adolescent direct self-injurious behavior: A comparative study of findings in 11 European countries. Journal of Child Psychology and Psychiatry 55, 4 (2014), 337-348.

[15] Jennifer Lee Carey, Brittany Chapman, Peter Chai, Danielle Jake-Schoffman, Stephanie Carreiro, Nathalie Nader, and Sherry Pagoto. 2018. SoMe and Self Harm: The use of social media in depressed and suicidal youth. In Proceedings of the 51st Hawaii International Conference on System Sciences.

[16] Yen-ning Chang, Youn-kyung Lim, and Erik Stolterman. 2008. Personas: from theory to practices. In Proceedings of the 5th Nordic conference on Human-computer interaction: building bridges. ACM, 439-442.

[17] Alexander L Chapman, Kim L Gratz, and Milton Z Brown. 2006. Solving the puzzle of deliberate self-harm: The experiential avoidance model. Behaviour research and therapy 44, 3 (2006), 371-394.

[18] Eun Kyoung Choe, Nicole B Lee, Bongshin Lee, Wanda Pratt, and Julie A Kientz. 2014. Understanding quantified-selfers' practices in collecting and exploring personal data. In Proceedings of the SIGCHI Conference on Human Factors in Computing Systems. ACM, 1143-1152.

[19] Annarosa Cipriano, Stefania Cella, and Paolo Cotrufo. 2017. Nonsuicidal self-injury: a systematic review. Frontiers in psychology 8 (2017), 1946.

[20] David Coyle and Gavin Doherty. 2009. Clinical Evaluations and Collaborative Design: Developing New Technologies for Mental Healthcare Interventions. In Proceedings of the SIGCHI Conference on Human Factors in Computing Systems (CHI '09). ACM, New York, NY, USA, 2051-2060. DOI : http://dx.doi.org/10.1145/1518701.1519013

[21] Kate Daine, Keith Hawton, Vinod Singaravelu, Anne Stewart, Sue Simkin, and Paul Montgomery. 2013. The power of the web: a systematic review of studies of the influence of the internet on self-harm and suicide in young people. PloS one 8, 10 (2013), e77555.

[22] Michele P Dyson, Lisa Hartling, Jocelyn Shulhan, Annabritt Chisholm, Andrea Milne, Purnima Sundar, Shannon D Scott, and Amanda S Newton. 2016. A systematic review of social media use to discuss and view deliberate self-harm acts. PloS one 11, 5 (2016), e0155813.

[23] Charlotte Emma Hilton. 2017. Unveiling self-harm behaviour: what can social media site Twitter tell us about self-harm? A qualitative exploration. Journal of clinical nursing 26, 11-12 (2017), 1690-1704. 
[24] Armando R Favazza. 1992. Repetitive self-mutilation. Psychiatric Annals 22, 2 (1992), 60-63.

[25] Joseph C Franklin, Kathryn R Fox, Christopher R Franklin, Evan M Kleiman, Jessica D Ribeiro, Adam C Jaroszewski, Jill M Hooley, and Matthew K Nock. 2016. A brief mobile app reduces nonsuicidal and suicidal self-injury: evidence from three randomized controlled trials. Journal of Consulting and Clinical Psychology 84, 6 (2016), 544.

[26] Madeleine George. 2019. The Importance of Social Media Content for Teens' Risks for Self-harm. Journal of Adolescent Health 65, 1 (2019), 9-10.

[27] Rebecca Grist, Joanna Porter, and Paul Stallard. 2018. Acceptability, Use, and Safety of a Mobile Phone App (BlueIce) for Young People Who Self-Harm: Qualitative Study of Service Users' Experience. JMIR mental health 5, 1 (2018), e16.

[28] Sarah Elisabeth Hetrick, Jo Robinson, Eloise Burge, Ryan Blandon, Bianca Mobilio, Simon M Rice, Magenta B Simmons, Mario Alvarez-Jimenez, Simon Goodrich, and Christopher G Davey. 2018. Youth codesign of a mobile phone app to facilitate self-monitoring and management of mood symptoms in young people with major depression, suicidal ideation, and self-harm. JMIR mental health 5, 1 (2018), e9.

[29] Hsiu-Fang Hsieh and Sarah E Shannon. 2005. Three approaches to qualitative content analysis. Qualitative health research 15, 9 (2005), 1277-1288.

[30] Stephen S Intille, AA Stone, and S Shiffman. 2007. Technological innovations enabling automatic, context-sensitive ecological momentary assessment. The science of real-time data capture: Self-reports in health research (2007), 308-337.

[31] Nina Jacob, Rhiannon Evans, and Jonathan Scourfield. 2017. The influence of online images on self-harm: A qualitative study of young people aged 16-24. Journal of adolescence 60 (2017), 140-147.

[32] Colleen M Jacobson and Madelyn Gould. 2007. The epidemiology and phenomenology of non-suicidal self-injurious behavior among adolescents: A critical review of the literature. Archives of Suicide Research 11, 2 (2007), 129-147.

[33] Ray Jones, Siobhan Sharkey, Janet Smithson, Tamsin Ford, Tobit Emmens, Elaine Hewis, Bryony Sheaves, and Christabel Owens. 2011. Using metrics to describe the participative stances of members within discussion forums. Journal of medical Internet research 13, 1 (2011), e3.

[34] Emily Klineberg, Stephen A Stansfeld, and Kamaldeep S Bhui. 2013. 119. How Do Adolescents Talk About Self-Harm? Findings From a Qualitative School-Based Study in England. Journal of Adolescent Health 52, 2 (2013), S78-S79.

[35] Brandin Hanson Knowles and Roisin McNaney. 2017. The Dark Side of Data Sharing: Considering the
Ethicality of Data Brokering within the Context of Health. Ethical Encounters in HCI (2017), 1-6.

[36] Lydia Manikonda and Munmun De Choudhury. 2017. Modeling and Understanding Visual Attributes of Mental Health Disclosures in Social Media. In Proceedings of the 2017 CHI Conference on Human Factors in Computing Systems (CHI '17). ACM, New York, NY, USA, 170-181. DOI :

http://dx.doi.org/10.1145/3025453.3025932

[37] Mark Matthews, Saeed Abdullah, Geri Gay, and Tanzeem Choudhury. 2014. Tracking mental well-being: Balancing rich sensing and patient needs. Computer 47 , 4 (2014), 36-43.

[38] Katelyn YA McKenna, Amie S Green, and Marci EJ Gleason. 2002. Relationship formation on the Internet: What's the big attraction? Journal of social issues 58, 1 (2002), 9-31.

[39] Lars Mehlum, Anita J Tørmoen, Maria Ramberg, Egil Haga, Lien M Diep, Stine Laberg, Bo S Larsson, Barbara H Stanley, Alec L Miller, Anne M Sund, and others. 2014. Dialectical behavior therapy for adolescents with repeated suicidal and self-harming behavior: a randomized trial. Journal of the American Academy of child \& adolescent psychiatry 53, 10 (2014), 1082-1091.

[40] Elizabeth M Miguel, Tommy Chou, Alejandra Golik, Danielle Cornacchio, Amanda L Sanchez, Mariah DeSerisy, and Jonathan S Comer. 2017. Examining the scope and patterns of deliberate self-injurious cutting content in popular social media. Depression and anxiety 34, 9 (2017), 786-793.

[41] Megan A Moreno, Adrienne Ton, Ellen Selkie, and Yolanda Evans. 2016. Secret society 123: Understanding the language of self-harm on Instagram. Journal of Adolescent Health 58, 1 (2016), 78-84.

[42] Jennifer J Muehlenkamp and others. 2014. Distinguishing between suicidal and nonsuicidal self-injury. The Oxford handbook of suicide and self-injury (2014), 23-46.

[43] Matthew K Nock. 2010. Self-injury. Annual review of clinical psychology 6 (2010), 339-363.

[44] Matthew K Nock and Armaruio R Favazza. 2009. Nonsuicidal self-injury: Definition and classification. (2009).

[45] Matthew K Nock, Thomas E Joiner Jr, Kathryn H Gordon, Elizabeth Lloyd-Richardson, and Mitchell J Prinstein. 2006. Non-suicidal self-injury among adolescents: Diagnostic correlates and relation to suicide attempts. Psychiatry research 144, 1 (2006), 65-72.

[46] Matthew K Nock, Mitchell J Prinstein, and Sonya K Sterba. 2009a. Revealing the form and function of self-injurious thoughts and behaviors: A real-time ecological assessment study among adolescents and young adults. Journal of abnormal psychology 118, 4 (2009), 816. 
[47] Matthew K Nock, Mitchell J Prinstein, and Sonya K Sterba. 2009b. Revealing the form and function of self-injurious thoughts and behaviors: A real-time ecological assessment study among adolescents and young adults. Journal of abnormal psychology 118, 4 (2009), 816.

[48] Justin W Patchin and Sameer Hinduja. 2017. Digital self-harm among adolescents. Journal of Adolescent Health 61, 6 (2017), 761-766.

[49] Jessica Pater and Elizabeth Mynatt. 2017. Defining Digital Self-Harm. In Proceedings of the 2017 ACM Conference on Computer Supported Cooperative Work and Social Computing (CSCW'17). ACM, New York, NY, USA, 1501-1513. DOI :

http://dx.doi.org/10.1145/2998181.2998224

[50] Jessica A. Pater, Oliver L. Haimson, Nazanin Andalibi, and Elizabeth D. Mynatt. 2016. "Hunger Hurts but Starving Works": Characterizing the Presentation of Eating Disorders Online. In Proceedings of the 19th ACM Conference on Computer-Supported Cooperative Work \& Social Computing (CSCW'16). ACM, New York, NY, USA, 1185-1200. DOI :

http://dx.doi.org/10.1145/2818048.2820030

[51] Jessica A. Pater, Andrew D. Miller, and Elizabeth D. Mynatt. 2015. This Digital Life: A Neighborhood-Based Study of Adolescents' Lives Online. In Proceedings of the 33rd Annual ACM Conference on Human Factors in Computing Systems (CHI '15). ACM, New York, NY, USA, 2305-2314. DOI :

http://dx.doi.org/10.1145/2702123.2702534

[52] Mashfiqui Rabbi, Shahid Ali, Tanzeem Choudhury, and Ethan Berke. 2011. Passive and In-Situ Assessment of Mental and Physical Well-being Using Mobile Sensors. In Proceedings of the 13th International Conference on Ubiquitous Computing (UbiComp '11). ACM, New York, NY, USA, 385-394. DOI : http://dx.doi.org/10.1145/2030112.2030164

[53] Stefan Rennick-Egglestone, Sarah Knowles, Gill Toms, Penny Bee, Karina Lovell, and Peter Bower. 2016. Health Technologies 'In the Wild': Experiences of Engagement with Computerised CBT. In Proceedings of the 2016 CHI Conference on Human Factors in Computing Systems (CHI '16). ACM, New York, NY, USA, 2124-2135. DOI :

http://dx.doi.org/10.1145/2858036.2858128

[54] Lucia Rodriguez-Blanco, Juan J Carballo, and Enrique Baca-Garcia. 2018. Use of ecological momentary assessment (EMA) in non-suicidal self-injury (NSSI): A systematic review. Psychiatry research 263 (2018), 212-219.

[55] Sarah L Rowe, Rebecca S French, Claire Henderson, Dennis Ougrin, Mike Slade, and Paul Moran. 2014. Help-seeking behaviour and adolescent self-harm: a systematic review. Australian \& New Zealand Journal of Psychiatry 48, 12 (2014), 1083-1095.
[56] Yukari Seko and Stephen P Lewis. 2018. The self-harmed, visualized, and reblogged: Remaking of self-injury narratives on Tumblr. New Media \& Society 20, 1 (2018), 180-198.

[57] Gail Slap, Elizabeth Goodman, and Bin Huang. 2001. Adoption as a risk factor for attempted suicide during adolescence. Pediatrics 108, 2 (2001), e30-e30.

[58] Sarah Stanford, Michael P Jones, and Jennifer L Hudson. 2017. Rethinking pathology in adolescent self-harm: Towards a more complex understanding of risk factors. Journal of adolescence 54 (2017), 32-41.

[59] stem4. 2019. Calm Harm App. (2019). http://calmharm.co.uk

[60] Lalita K Suzuki and Jerel P Calzo. 2004. The search for peer advice in cyberspace: An examination of online teen bulletin boards about health and sexuality. Journal of applied developmental psychology 25, 6 (2004), 685-698.

[61] Melanie Swan. 2009. Emerging patient-driven health care models: an examination of health social networks, consumer personalized medicine and quantified self-tracking. International journal of environmental research and public health 6, 2 (2009), 492-525.

[62] Lindsay A Taliaferro and Jennifer J Muehlenkamp. 2014. Risk and protective factors that distinguish adolescents who attempt suicide from those who only consider suicide in the past year. Suicide and Life-Threatening Behavior 44, 1 (2014), 6-22.

[63] Hiran Thabrew, Theresa M Fleming, Sarah Hetrick, and Sally Nicola Merry. 2018. Co-design of eHealth interventions with children and young people. Frontiers in psychiatry 9 (2018), 481.

[64] Anja Thieme, John McCarthy, Paula Johnson, Stephanie Phillips, Jayne Wallace, Siân Lindley, Karim Ladha, Daniel Jackson, Diana Nowacka, Ashur Rafiev, Cassim Ladha, Thomas Nappey, Mathew Kipling, Peter Wright, Thomas D. Meyer, and Patrick Olivier. 2016. Challenges for Designing New Technology for Health and Wellbeing in a Complex Mental Healthcare Context. In Proceedings of the $2016 \mathrm{CHI}$ Conference on Human Factors in Computing Systems (CHI'16). ACM, New York, NY, USA, 2136-2149. DOI : http://dx.doi.org/10.1145/2858036.2858182

[65] Joseph Tighe, Fiona Shand, Rebecca Ridani, Andrew Mackinnon, Nicole De La Mata, and Helen Christensen. 2017. Ibobbly mobile health intervention for suicide prevention in Australian Indigenous youth: a pilot randomised controlled trial. BMJ open 7, 1 (2017), e013518.

[66] John Torous, Mathew V Kiang, Jeanette Lorme, and Jukka-Pekka Onnela. 2016. New tools for new research in psychiatry: a scalable and customizable platform to empower data driven smartphone research. JMIR mental health 3, 2 (2016), e16. 
[67] John Torous, Mark E Larsen, Colin Depp, Theodore D Cosco, Ian Barnett, Matthew K Nock, and Joe Firth. 2018. Smartphones, sensors, and machine learning to advance real-time prediction and interventions for suicide prevention: a review of current progress and next steps. Current psychiatry reports 20, 7 (2018), 51.

[68] Ellen Townsend, Ruth Wadman, Kapil Sayal, M Armstrong, C Harroe, P Majumder, Panayotis Vostanis, and David Clarke. 2016. Uncovering key patterns in self-harm in adolescents: Sequence analysis using the Card Sort Task for Self-harm (CaTS). Journal of affective disorders 206 (2016), 161-168.

[69] Rui Wang, Weichen Wang, Min SH Aung, Dror Ben-Zeev, Rachel Brian, Andrew T Campbell, Tanzeem Choudhury, Marta Hauser, John Kane, Emily A Scherer, and others. 2017b. Predicting symptom trajectories of schizophrenia using mobile sensing. Proceedings of the ACM on Interactive, Mobile, Wearable and Ubiquitous Technologies 1, 3 (2017), 110.

[70] Yilin Wang, Jiliang Tang, Jundong Li, Baoxin Li, Yali Wan, Clayton Mellina, Neil O'Hare, and Yi Chang. 2017a. Understanding and Discovering Deliberate Self-harm Content in Social Media. In Proceedings of the 26th International Conference on World Wide Web ( $W W W$ '17). International World Wide Web Conferences Steering Committee, Republic and Canton of Geneva, Switzerland, 93-102. DOI :

http://dx.doi.org/10.1145/3038912.3052555

[71] Janis Whitlock, John Eckenrode, and Daniel Silverman. 2006. Self-injurious behaviors in a college population. Pediatrics 117, 6 (2006), 1939-1948.

[72] Janis Whitlock, Jennifer Muehlenkamp, John Eckenrode, Amanda Purington, Gina Baral Abrams, Paul Barreira, and Victoria Kress. 2013. Nonsuicidal self-injury as a gateway to suicide in young adults. Journal of Adolescent Health 52, 4 (2013), 486-492.

[73] Katrina Witt, Matthew J Spittal, Gregory Carter, Jane Pirkis, Sarah Hetrick, Dianne Currier, Jo Robinson, and Allison Milner. 2017. Effectiveness of online and mobile telephone applications ('apps') for the self-management of suicidal ideation and self-harm: a systematic review and meta-analysis. BMC psychiatry 17, 1 (2017), 297. 